\title{
Hemifield-specific Correlations between Cue-related Blood Oxygen Level Dependent Activity in Bilateral Nodes of the Dorsal Attention Network and Attentional Benefits in a Spatial Orienting Paradigm
}

Citation for published version (APA):

Mayrhofer, H. C., Duecker, F., van de Ven, V., Jacobs, H. I. L., \& Sack, A. T. (2019). Hemifield-specific Correlations between Cue-related Blood Oxygen Level Dependent Activity in Bilateral Nodes of the Dorsal Attention Network and Attentional Benefits in a Spatial Orienting Paradigm. Journal of Cognitive Neuroscience, 31(5), 625-638. https://doi.org/10.1162/jocn_a_01338

Document status and date:

Published: 01/05/2019

DOI:

10.1162/jocn_a_01338

Document Version:

Publisher's PDF, also known as Version of record

Document license:

Taverne

Please check the document version of this publication:

- A submitted manuscript is the version of the article upon submission and before peer-review. There can be important differences between the submitted version and the official published version of record. People interested in the research are advised to contact the author for the final version of the publication, or visit the DOI to the publisher's website.

- The final author version and the galley proof are versions of the publication after peer review.

- The final published version features the final layout of the paper including the volume, issue and page numbers.

Link to publication

\footnotetext{
General rights rights.

- You may freely distribute the URL identifying the publication in the public portal. please follow below link for the End User Agreement:

www.umlib.nl/taverne-license

Take down policy

If you believe that this document breaches copyright please contact us at:

repository@maastrichtuniversity.nl

providing details and we will investigate your claim.
}

Copyright and moral rights for the publications made accessible in the public portal are retained by the authors and/or other copyright owners and it is a condition of accessing publications that users recognise and abide by the legal requirements associated with these

- Users may download and print one copy of any publication from the public portal for the purpose of private study or research.

- You may not further distribute the material or use it for any profit-making activity or commercial gain

If the publication is distributed under the terms of Article 25fa of the Dutch Copyright Act, indicated by the "Taverne" license above,

Download date: 26 Apr. 2023 


\title{
Hemifield-specific Correlations between Cue-related Blood Oxygen Level Dependent Activity in Bilateral Nodes of the Dorsal Attention Network and Attentional Benefits in a Spatial Orienting Paradigm
}

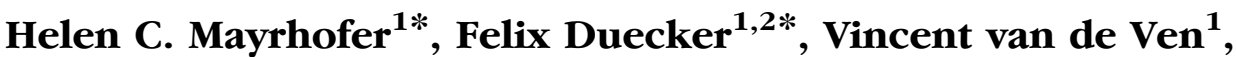 \\ Heidi I. L. Jacobs ${ }^{1,3}$, and Alexander T. Sack ${ }^{1}$
}

\begin{abstract}
The dorsal attention network (DAN) is known to be involved in shifts of spatial attention or in orienting. However, the involvement of each hemisphere in shifts to either hemifield is still a matter of debate. In this study, interindividual hemifield-specific attentional benefits in RTs were correlated with cue-related BOLD responses specific to directive cues in the left and right frontal and posterior nodes of the DAN, measured in a Spatial Orienting Paradigm. The pattern of correlations was analyzed with respect to its fit with three existing hypotheses of spatial attention control: the contralateral, right dominance, and hybrid hypotheses. Results showed that activation in frontal and parietal nodes of the DAN could explain a significant proportion of the interindividual variance in attentional benefits. Although
\end{abstract}

\section{INTRODUCTION}

As we navigate the world, our visual system is confronted with a seemingly endless stream of inputs that compete for our limited processing capacities. "Spatial attention," the ability to scan the environment and select behaviorally relevant stimuli at specific spatial locations for enhanced processing, is considered one of the most fundamental mechanisms enabling us to interact with our environment in a goal-directed manner (James, 1890).

First evidence for the neural underpinnings of spatial attention could be gathered from studying patients experiencing hemispatial neglect as a consequence of brain damage. These patients fail to respond to stimuli presented in the hemifield contralateral to the brain lesion, and it appears that this particular behavior stems from an attentional deficit (Posner, Walker, Friedrich, \& Rafal, 1987; Mesulam, 1981). This suggests that each hemisphere is involved in shifts of attention to the contralateral hemifield (Kinsbourne, 1970).

\footnotetext{
${ }^{1}$ Maastricht University, ${ }^{2}$ University of Coimbra, ${ }^{3}$ Massachusetts General Hospital/Harvard Medical School

*Shared first authorship.
}

However, this simple notion cannot explain the finding that hemispatial neglect tends to be more common and more severe if the right hemisphere is affected as opposed to the left hemisphere (e.g., Suchan, Rorden, \& Karnath, 2012). Different explanations for these hemispheric asymmetries in neglect pathology have been offered, one prominent one proposing that, whereas the left hemisphere exclusively mediates attention shifts to the right hemifield, the right hemisphere can mediate attention shifts to both hemifields and can thus compensate for damage in the left hemisphere (Mesulam, 1981; Heilman \& Van den Abell, 1980; Heilman \& Valenstein, 1979; but see also Kinsbourne, 1970, for a competing explanation, and Bartolomeo \& Chokron, 1999, for a direct comparison of theories).

Although this and other contemporary theories of attention often spoke of the entire hemisphere as the entity involved in attention shifts, or focused on the parietal lobe as the locus of attention, neurophysiological studies in animals and neuroimaging studies in humans conducted over the past decades have allowed us to identify a number of specific cortical and subcortical regions supporting the orienting of attention (Kastner \& Ungerleider, 2000; 
Desimone \& Duncan, 1995). Among these regions, a frontoparietal network comprising parts of the intraparietal sulcus (IPS) extending into the superior parietal lobe (SPL) and the frontal eye fields (FEFs) has been ascribed a prominent role in the voluntary orienting and maintenance of attention. This network has been labeled the dorsal attention network (DAN; Corbetta \& Shulman, 2002).

Substantial research has been conducted to determine the exact role that the DAN plays in attention processes in particular and cognition in general (see Lückmann, Jacobs, \& Sack, 2014, for a review). Many of the tasks used involve some form of spatially informative cue predicting the location of an upcoming target, thereby prompting participants to orient their attention to the cued location (Chica, Martín-Arévalo, Botta, \& Lupiáñez, 2014; Posner, 1980). Conventional fMRI group analyses suggest that spatial cues to the left and to the right activate the bilateral DAN, but hemispheric preferences for cues pointing to the contralateral hemifield have also been detected (Shulman et al., 2009, 2010; Sylvester, Shulman, Jack, \& Corbetta, 2007), which is in line with the assumption that each hemisphere primarily coordinates shifts of attention to the contralateral hemifield. With respect to hemispheric asymmetries, Shulman et al. (2010) could not find stronger cue-related activation for ipsilateral cues in the right-hemispheric DAN compared with the left, as would be expected in case the right hemisphere indeed mediates attention shifts to the left, as well as to the right, visual hemifield. The authors took this as evidence that right-hemispheric dominance occurs in brain regions outside the DAN and that the relationship between DAN activation and attention shifts to the left and right hemifields is predominantly contralateral (see also Corbetta \& Shulman, 2011).

Yet, a different pattern may emerge when correlating cue-related DAN activation and behavioral effects in the context of spatial orienting. These correlations make use of the information that is contained in the variability of the neural and behavioral responses (between trials or between individuals) and may thus be more sensitive when it comes to disentangling the involvement of a particular region in a task. So far, only few studies have attempted to link brain activation outside the visual cortex during spatial attention tasks to behavioral effects of spatial orienting (Sylvester et al., 2007; Sapir, D'Avossa, McAvoy, Shulman, \& Corbetta, 2005; Small et al., 2003). Sapir and colleagues found a difference in cue-related activation in posterior DAN regions between correct and incorrect trials that depended on cue validity (Sapir et al., 2005), whereas Sylvester et al. (2007) found that, although the difference in activation between the two hemispheres could predict accuracies in a hemifieldspecific manner, suggesting contralateral preferences, this applied to regions in the visual cortex only. Similarly, Small et al. (2003) reported differences in activation mostly outside the DAN between trials with strong behavioral effects of orienting and those without. These fMRI studies of spatial orienting have not been able to reveal behaviorally relevant patterns of activation in the DAN that can account for the asymmetries observed in neglect pathology, and they often failed to find neural correlates of behavioral effects of attention in the DAN altogether.

However, these studies might have failed to find correlates in the DAN because their design did not properly allow for the isolation of attentional effects, especially with respect to behavioral measures (Sylvester et al., 2007; Sapir et al., 2005), or because they did not separate cue-related activation from that related to target processing (Small et al., 2003). More importantly, only one of these studies tested directly for differences between the two hemispheres in a hemifield-specific manner (Sylvester et al., 2007), but the study design did not allow for the detection of a potential righthemispheric dominance.

In this study, we aimed to assess whether we could uncover a role of the DAN in attentional processes by using a design that was tailored to the detection of interindividual differences in orienting-specific effects on behavior and brain activation in the DAN. More specifically, we measured attentional benefits, the average difference in RTs in response to targets preceded by a valid spatial cue compared with targets preceded by a spatially uninformative neutral cue, and we correlated these attentional benefits with an estimate of the average cuerelated BOLD response specific to spatially informative cues as opposed to neutral cues in the DAN. The subtraction of the neutral condition in both measures allowed us to isolate brain activation and behavioral effects specific to spatial attention shifts. Furthermore, the estimate of DAN activation was obtained from catch trials without a target, thus ensuring that the recorded BOLD response was anticipatory in nature and not influenced by target processing. Correlations were calculated across individuals in a sample of 28 participants. To be sensitive toward potential cue-specific differences between the regions of the DAN (particularly between the left and right hemispheres), correlations were calculated separately for left and right cues and for left and right FEF and IPS/SPL, localized for each participant individually.

In a second step, we wanted to test explicitly whether the observed pattern of correlations across the four nodes of the DAN fits with any of three hypotheses derived from existing models of attention control: The first hypothesis predicts that each region of the DAN is involved in shifts of attention to the contralateral hemifield only (contralateral hypothesis; Corbetta \& Shulman, 2011; Shulman et al., 2010; Kinsbourne, 1970), the second predicts that activation in the right-hemispheric DAN additionally correlates with benefits in the right hemifield (right dominance; Mesulam, 1981; Heilman \& Van den Abell, 1980; Heilman \& Valenstein, 1979), and the third dissociates between frontal and parietal regions 
of the DAN and predicts right-hemispheric dominance in FEF only and a purely contralateral relationship between brain activation and behavior in IPS/SPL, in line with a recent review of findings from brain stimulation studies (hybrid; Duecker \& Sack, 2015).

\section{METHODS}

\section{Sample}

Thirty-three participants (19 women) aged 18-37 years were included in the study. One participant was lefthanded, the rest were right-handed, and all participants had normal or corrected-to-normal vision and were fluent in the English language. Procedures were approved by the institutional review board of the Faculty of Psychology and Neuroscience of Maastricht University. Written informed consent was obtained from all participants as part of the magnetic resonance screening procedure. Participants received course credit or $€ 10$ per hour as compensation. Two participants stopped their participation prematurely because of discomfort inside the MRI scanner, two participants were excluded from the analyses because they performed the spatial attention task with low accuracy compared with the rest of the sample, and one participant was excluded because of excessive motion during MRI data acquisition. The final sample included in the analyses comprised 28 participants (15 women; age: $M=$ $25.57, S D=4.41)$.

\section{Tasks and Stimulus Presentation}

During MRI acquisition, the visual display was projected onto a screen inside the scanner bore with a resolution of $1920 \times 1200$ pixels and a diagonal of $18.5 \mathrm{in}$. (47 cm). Participants could see this display through a mirror attached to the head coil. The distance between the screen and the mirror $(\sim 60 \mathrm{~cm})$ and the distance between the mirror and the eyes of the participants $(\sim 15 \mathrm{~cm})$ amounted to approximately $75 \mathrm{~cm}$.

\section{DAN Localizer}

To account for the substantial individual variability in anatomy and functional organization in the association cortex, we chose to localize participant-specific ROIs in the DAN based on functionally defined brain maps. To this end, we used a localizer for regions involved in overt shifts of attention. Neuroimaging studies suggest that clusters of activation for overt and covert shifts of attention are largely overlapping (de Haan, Morgan, \& Rorden, 2008; Nobre, Gitelman, Dias, \& Mesulam, 2000), and brain stimulation studies using a similar localizer for the stimulation sites have reported effects on spatial attention (Duecker, Formisano, \& Sack, 2013). Participants were instructed to make eye movements to follow a moving dot presented to them on the screen inside the scanner. The dot, a filled white circle with a diameter of $0.5^{\circ}$ of visual angle, was presented on a black background and could appear at one of nine possible locations along the vertical and horizontal meridians: central or $5^{\circ}$ or $8^{\circ}$ of visual angle left/right or up/down from the center. The BOLD response related to eye movements was assessed in a blocked design, with alternating 20-sec blocks of fixation, during which the dot stayed at the central location, and 13-sec blocks of eye movement, during which the dot was displayed at the peripheral locations, changing location every $600 \mathrm{msec}$ in a random order (22 location changes). Eleven fixation blocks and 10 eye movement blocks were presented. The routine was controlled with the Presentation software package (Neurobehavioral Systems).

\section{Spatial Orienting Paradigm}

Spatial orienting performance and DAN activation during shifts of spatial attention were assessed in a covert endogenous (i.e., goal-driven) variant of the Spatial Orienting Paradigm with a discrimination task (Chica et al., 2014; Posner, 1980). In this paradigm, central symbolic spatial cues inform participants about the likely location of an upcoming target, to which participants have to respond while keeping their eyes on a central fixation point. It has been shown that participants use the spatial information provided by the cue to direct their attention to the cued location and respond faster on average if the target appears in the cued location (valid trials) and slower if the target appears in the uncued location (invalid trials) compared with trials in which the cue conveys no spatial information (neutral trials; Posner, 1980). Attentional benefits, the difference in average RTs between valid and neutral trials, can be used to quantify a participant's spatial orienting response, which is thought to depend upon DAN functionality.

In the present variant of the task, targets were Gabor patches (spatial frequency $=1.5$ cycles per degree, envelope $S D=0.66^{\circ}$, Michelson contrast $=50 \%$, random phase) presented left or right of the center of a mean gray screen (marked by a black-and-white bull's-eye) at an eccentricity of $7^{\circ}$ of visual angle. Target orientation was either $45^{\circ}$ or $135^{\circ}$ (with a random jitter of up to $5^{\circ}$ in each direction), and participants had to indicate target orientation by pressing one of two adjacent response buttons using the index and middle fingers of their right hand. Pairs of arrows presented both left and right of fixation in close proximity to the bull's-eye $\left(1.5^{\circ}\right)$ were used as spatial cues. Cues could be neutral (arrows on both sides pointing to the periphery and thus to opposite hemifields) or directive (arrows on both sides pointing to the same hemifield). Participants were told that targets were equally likely to appear in either hemifield after a neutral cue and were more likely to appear in the cued hemifield after a directive cue. The inclusion of neutral trials allowed us not only to calculate attentional benefits but also to contrast BOLD responses for directive and 
neutral cues to single out neural mechanisms that are specific to spatial orienting as opposed to more general cue-related responses due to, for example, visual input or temporal information.

Cues were presented for $100 \mathrm{msec}$, followed by a variable interval of $2.1,3.2$, or $4.3 \mathrm{sec}$, after which the target was displayed for $100 \mathrm{msec}$. Then, $900 \mathrm{msec}$ after target offset, the outer circle of the fixation bull's-eye turned gray, which marked the onset of a variable intertrial interval of $10,12.2$, or $14.4 \mathrm{sec}$. Participants' responses to targets were recorded until 2000 msec after target onset (i.e., 1000 msec into the intertrial interval), but participants were instructed to respond as fast as possible without making mistakes.

Importantly, the task also included catch trials, during which no target was presented. Trial timings were the same as for the trials with a target except that no target was presented in the $100 \mathrm{msec}$ after the delay interval, thus allowing for the measurement of a cue-related BOLD response that is not biased by target-related activation (see Figure 1 for a schematic of the sequence of events per trial type). Participants performed 252 trials, divided into six runs of 42 trials each. One hundred forty-four of the trials included a directive cue followed by a target. On $75 \%$ of these trials, the cue correctly predicted the location of the target (108 valid trials and 36 invalid trials). Although we were interested in attentional benefits only, invalid trials were included to decrease participants' tendencies to make eye movements toward the cued hemifield. The remaining trials were equally composed of neutral trials with a target, neutral catch trials, and directive catch trials (36 trials each, 18 per cued hemifield). The ratio of trial types within a run corresponded to the overall ratio across all trials (3:1:1:1:1), and target location and cue target intervals were balanced within each trial type within a run. The order of trials within a run was randomized, and the task was controlled with PsychoPy (v1.80.04; Peirce, 2008).

\section{MRI Acquisition and Preprocessing}

Imaging data were acquired on a Magnetom Prisma 3-T MRI scanner with a 32-channel head coil (Siemens). A high-resolution anatomical image was obtained using a sagittal MPRAGE sequence (repetition time $[\mathrm{TR}]=2250 \mathrm{msec}$, echo time $=2.21 \mathrm{msec}$, flip angle $=9^{\circ}$, voxel size $=1 \mathrm{~mm}$ isotropic, field of view $=256 \mathrm{~mm}, 192$ slices). The BOLD response was measured with an axial oblique gradientecho EPI sequence with a multiband acceleration factor of $2\left(\mathrm{TR}=1100 \mathrm{msec}\right.$, echo time $=30 \mathrm{msec}$, flip angle $=64^{\circ}$, voxel size $=3 \mathrm{~mm}$ isotropic, field of view $=216 \mathrm{~mm}$, 34 slices, interleaved slice acquisition). The slice box was adjusted to cover most of the cerebrum with the exception of orbitofrontal regions for some participants. Data were preprocessed using BrainVoyager QX for Windows, Version 2.8.4 (Brain Innovation B.V.; Goebel, 2012). Preprocessing included slice scan time correction, intrarun and interrun volume alignment to the first volume after the MPRAGE to correct for head motion, and high-pass temporal filtering using a general linear model (GLM) approach (two cycles) including linear trend removal. Functional data of each participant were coregistered to the anatomical image using a nine-parameter fine alignment approach and were subsequently transformed to Talairach space.

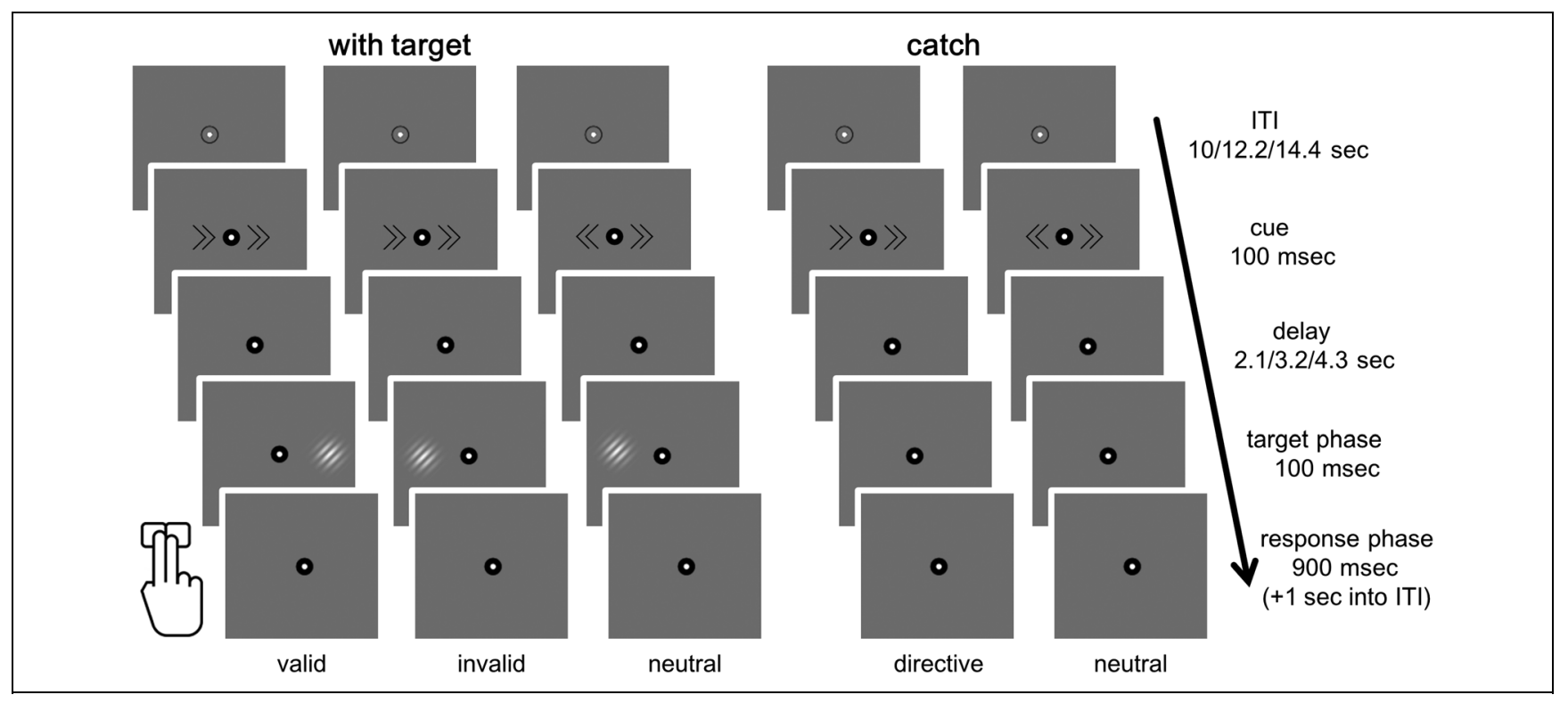

Figure 1. Spatial Orienting Paradigm: trial types. Examples display only a selection of possible trials and are not to scale (please refer to the text for actual dimensions and locations). All possible combinations of cued hemifield (left, right, both) and target hemifield (left, right) per condition were presented to participants. ITI $=$ intertrial interval. 


\section{Experimental Procedure}

At least 2 days before the actual MRI session, all participants were invited for a short training to familiarize them with the Spatial Orienting Paradigm. The training session served to confirm that participants understood the task and were able to refrain from moving their eyes away from fixation during task performance (assessed by the experimenter based on observation).

At the beginning of the scanning session, an 8-min resting state scan (436 volumes) was obtained. Participants were asked to keep their eyes open and to fixate on a blackand-white bull's-eye presented at the center of a mean gray screen throughout this scan. These data were collected for a different research purpose and not further analyzed in the context of this study. Afterward, the DAN localizer was run during a 6-min functional scan (317 volumes). Subsequently, orienting performance and brain activation were measured in six functional task runs of approximately $12 \mathrm{~min}$ (661 volumes), with a break after three runs to acquire the high-resolution anatomical image $(5 \mathrm{~min})$.

\section{Behavioral Data Analysis}

Data cleaning and aggregation were completed with MATLAB (MATLAB Release 2013a, The MathWorks, Inc.). Before averaging RTs per condition and participant, data from incorrect trials and outliers (RTs more than 1.5 times the interquartile range above the 75th or below the 25th percentile for that condition and individual) were removed. Two participants performed the task with low accuracy compared with the rest of the sample and were excluded from further analyses. For the remaining participants, average RTs of all conditions were based on at least 11 trials and the average accuracy was $95.1 \%$. RT data were entered in a $2 \times 3$ repeated-measures ANOVA with factors Target hemifield (left, right) and Cue (valid, neutral, invalid) to confirm that cue validity had an effect on RTs and to assess whether this effect depended on target hemifield. We tested for violations of the assumption of sphericity (Mauchly, 1940) and adjusted the degrees of freedom accordingly (Greenhouse \& Geisser, 1959). To confirm that cues affected RTs in the expected direction, paired $t$ tests were conducted to compare average RTs between valid and neutral trials (attentional benefits) and invalid and neutral trials (attentional costs). Partial $\eta^{2}\left(\eta_{\mathrm{p}}^{2}\right)$ was obtained as an estimate of effect size for $F$ tests (Cohen, 1973), and Cohen's $d_{\mathrm{z}}$ was calculated as an estimate of effect size for paired $t$ tests (Cohen, 1988, p. 48). The ANOVA was run using the Statistical Package for the Social Sciences (SPSS; IBM SPSS Statistics for Windows, Version 21; IBM Corp.).

\section{fMRI Data Analysis}

Selection of ROIs Based on DAN Localizer

ROIs in the left and right FEFs and IPS/SPL were defined for each participant individually, based on the DAN localizer. In a first step, we obtained single-subject statistical ( $t$ value) maps contrasting brain activation during eye movement to that during fixation, using BrainVoyager to run a simple GLM with a single boxcar predictor of eye movement periods, convolved with a standard two-gamma hemodynamic response function. These maps were overlaid onto the individual structural volume to identify the most prominent cluster of 10 functional active voxels in each hemisphere in the vicinity of the intersection of the superior frontal sulcus and the precentral sulcus that marks the FEF (Petit \& Haxby, 1999).

To localize the IPS/SPL nodes of the DAN for each participant, we looked not only at activation related to eye movement, which is fairly widespread in IPS/SPL, but also at the functional connectivity with FEF during eye movement, to restrict the ROIs to those regions that appear to form a functionally connected network. To do so, the average time series of the fMRI signal during the DAN localizer was extracted and $z$-transformed for each selected FEF ROI (using NeuroElf for MATLAB, www. neuroelf.net) and was then entered as a predictor in a GLM with the task predictor for active eye movement blocks entered as an additional confound. The resulting two statistical maps obtained per participant, which respectively reflected the functional connectivity with the left and right FEFs, were averaged and finally masked with a binary mask of voxels that were active during eye movement compared with baseline, thresholded at $q=.05$ controlling the false discovery rate (FDR; Benjamini \& Hochberg, 1995). We then manually selected 10 continuous functional voxels displaying high average connectivity with FEF in the vicinity of the seed coordinate for the right IPS/SPL $(27,-58,49)$ used by Fox, Corbetta, Snyder, Vincent, and Raichle (2006), which was based on their meta-analysis of five fMRI studies of spatial orienting, and defined those as the right IPS/SPL ROI on the individual level. For left IPS/SPL, we selected the cluster of 10 voxels with high average connectivity with FEF that most closely mirrored the right IPS/SPL ROI. For one participant, the task-based mask turned out to be too restrictive and the threshold was adjusted to a level of $p=.05$, uncorrected.

Figure 2 shows the location and overlap of ROIs across participants on the mean structural MRI of the group, and Table 1 provides coordinates of a central voxel shared by the highest number of participants for each ROI. We also provide information on the cytoarchitectonic region that each peak voxel falls within most probably according to the Jülich cytoarchitectonic atlas (Eickhoff et al., 2007). To determine this, we transformed the peak coordinates to Montreal Neurological Institute space using an online tool (sprout022.sprout.yale.edu/mni2tal/mni2tal.html; Lacadie, Fulbright, Constable, \& Papademetris, 2008) and then used the FMRIB Software Library (v5.0) to query the atlas information (Jenkinson, Beckmann, Behrens, Woolrich, \& Smith, 2012). 
Figure 2. Distribution and overlap of ROIs across participants. Colors represent the number of participants sharing the voxel in the respective ROI. ROIs are projected on the mean structural MRI of the group. Coordinates at the slice intersection mark a peak voxel in the ROI for right IPS/SPL.

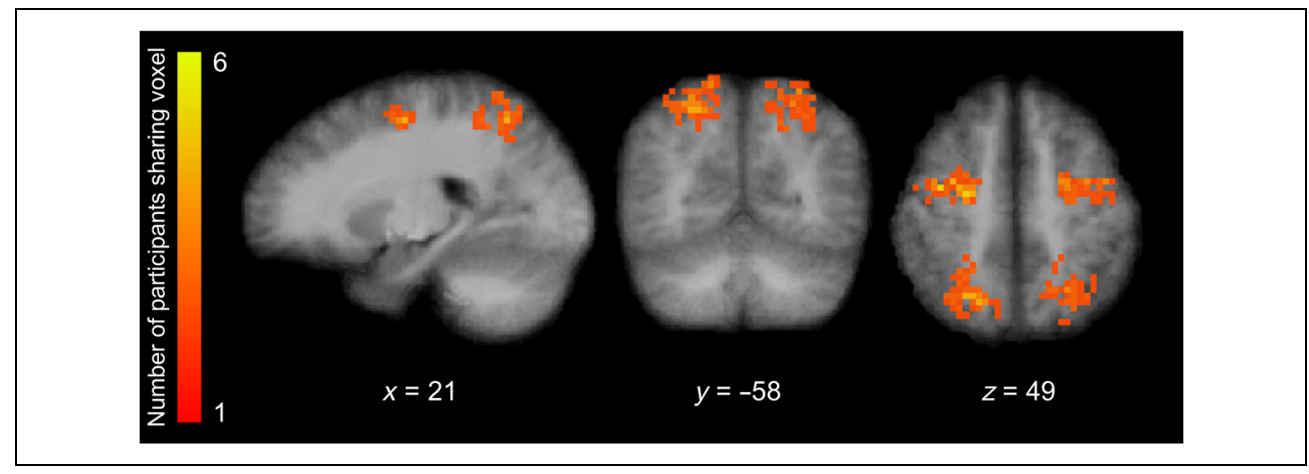

\section{GLM Analysis and Cue-related BOLD Response}

To model the BOLD response during the Spatial Orienting Paradigm, we created separate boxcar predictors for neutral cues and left and right cues with and without a target (six cue predictors) and for valid, invalid, and neutral targets (three target predictors). Cue predictors were modeled from cue onset to target onset for trials with a target and extended by another TR for catch trials. Target predictors were modeled for one TR from target onset onward. Target trials for which participants gave no response (omission errors) were modeled separately with a separate predictor for cue and target and included as confounds, because it is unclear why participants failed to respond to these trials (drowsiness, eye blinks, etc.). Target trials with incorrect responses (i.e., misidentifying the target orientation) were not treated differently. All predictors were convolved with a twogamma hemodynamic response function and were included in a random effects GLM to obtain individual beta maps for each predictor per participant. In doing so, voxel-wise data were normalized to reflect the percent signal change from the mean signal across time, and data were prewhitened assuming a second-order autoregressive process to correct for serial correlation.

For each ROI, individual betas for the contrasts between catch trials with a leftward cue and those with a neutral cue and between catch trials with a rightward cue and those with a neutral cue were extracted using NeuroElf, resulting in eight betas (two per ROI). To test

Table 1. Peak Voxel Locations and Cytoarchitectonic Labels of ROIs

\begin{tabular}{lcl}
\hline ROI & TAL Coordinates & Cytoarchitectonic Region \\
\hline Left FEF & $-24,-7,43$ & Premotor cortex (BA 6 L) \\
Right FEF & $24,-10,49$ & Premotor cortex (BA 6 R) \\
Left IPS/SPL & $-27,-61,55$ & SPL (7A L) \\
Right IPS/SPL & $21,-58,49$ & SPL (7A R) \\
\hline
\end{tabular}

Voxel coordinates refer to a voxel shared by the highest number of participants in each ROI; cytoarchitectonic regions are regions the voxel most probably falls within according to the Jülich cytoarchitectonic atlas (Eickhoff et al., 2007). BA = Brodmann's area; TAL $=$ Talairach whether ROIs generally responded specifically to the directive cues, one-sided one-sample $t$ tests of the obtained betas per ROI and contrast were computed (in SPSS). Furthermore, the betas were entered in a $2 \times 2 \times 2$ within-subject ANOVA with factors Cue (left, right), ROI (FEF, IPS/SPL), and Hemisphere (left, right).

\section{Correlation Analyses}

Individual hemifield-specific attentional benefits were calculated by computing the difference in average RTs between the neutral cue condition and the valid cue condition (in milliseconds) for targets presented in the left and right hemifields. Individual benefits in the left (right) hemifield were then correlated with the abovementioned betas for the contrast of brain activation for catch trials with left (right) cues with catch trials with neutral cues from each of the four ROIs, resulting in eight correlations reflecting the degree to which individual cue-related activation (for left and right cues) in the FEF and IPS/SPL nodes of the DAN can explain interindividual variance of attentional benefits in the two hemifields. In keeping with existing hypotheses of contralateral preference, we expected high positive correlations between brain activation and contralateral benefits in all regions. With respect to right-hemispheric dominance, we also expected a high correlation between right benefits and brain activation in the right FEF. These correlations were thus tested onesided. In keeping with all existing hypotheses of attention control, we expected no correlations between activation in the left FEF and IPS/SPL and benefits in the left hemifield and ran two-sided tests for these correlations as well as the correlation between activation in the right IPS/SPL and benefits in the right hemifield, as findings from brain stimulation studies suggest that the right IPS/SPL does not play a role for attention shifts to the right hemifield (Duecker \& Sack, 2015). All resulting $p$ values were FDR-corrected.

\section{Permutation Tests of Hypotheses}

Next to the effect size and significance level of the individual correlations, we were also interested in the specific pattern of correlations and the extent to which the pattern fitted predictions made by existing hypotheses of 
Figure 3. Effect of cue validity on RTs. Colored lines represent individual RTs per cue condition. Circles mark mean $\mathrm{RTs}$, and vertical black lines mark the SEM per cue condition. $* p<.001$.

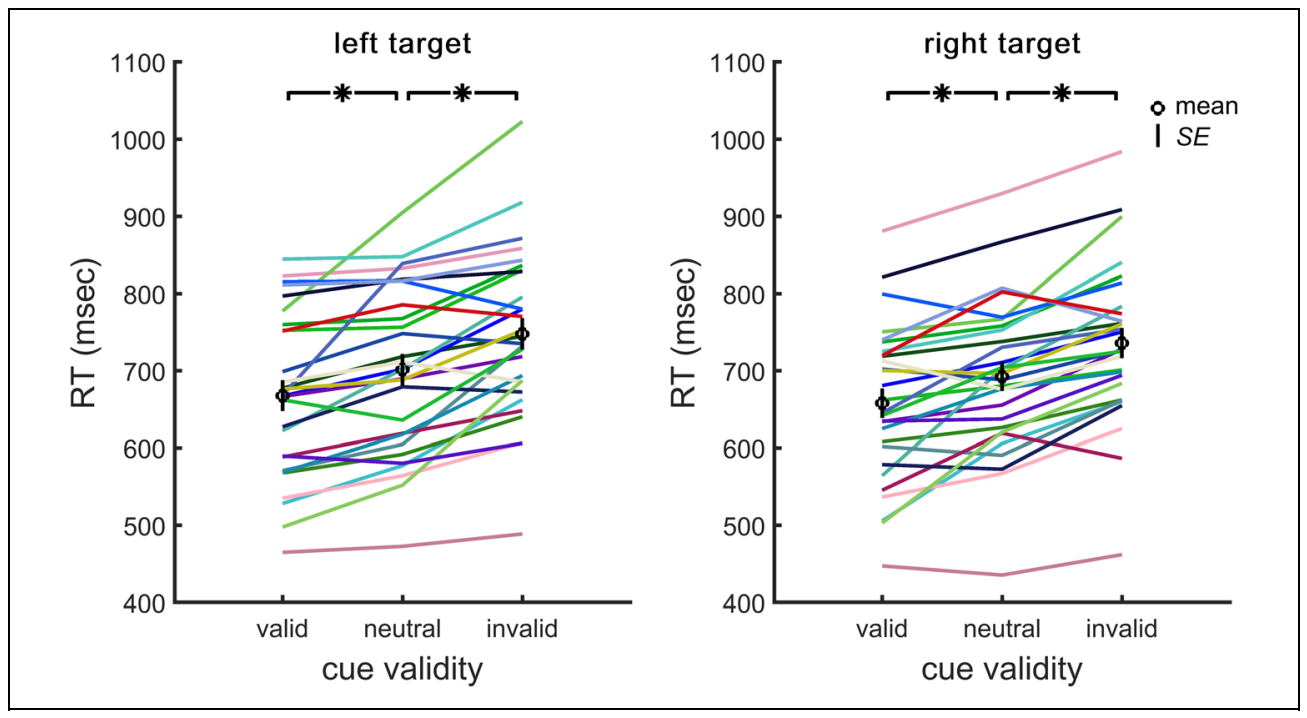

attention control. To investigate this issue, we split the eight correlations into two groups of expected high and expected low correlations in accordance with each of the three alternative hypotheses (see Table 3 for groupings per hypothesis). We then transformed each correlation with Fisher's $r$-to- $z$ transformation and calculated the mean difference between the expected low and expected high correlations. To test the significance of the obtained differences, we tested them against nonparametric test distributions assuming that the correlations are in fact all equal (i.e., the observed differences between correlations are due to noise) by permuting the eight betas per participant $(1,000,000$ permutations) to then recalculate the eight $z$-transformed correlations and the difference between expected high and low correlations per permutation. The percentages of cases in which the randomly generated pattern resulted in an equal or greater mean difference between the two groups of correlations compared with the actual observed pattern of correlations were taken as an estimate of the probability of obtaining the observed "hypothesis fit" given the distribution of individual betas.

\section{RESULTS}

\section{Behavioral Data}

We found a significant main effect of Cue, $F(1.39,37.44)=$ 49.49, $p<.001, \eta_{\mathrm{p}}^{2}=.647$, and no main effect of Hemifield, $F(1,27)=1.26, p=.271, \eta_{\mathrm{p}}^{2}=.045$, or interaction of Hemifield and Cue, $F(2,54)=0.06, p=.946$, $\eta_{\mathrm{p}}^{2}=.002$. Pairwise comparisons of cue conditions revealed that RTs were significantly faster on valid trials compared with neutral trials (mean attentional benefits $=34 \mathrm{msec}$, $S D=34 \mathrm{msec}), t(27)=5.26, p<.001, d_{\mathrm{z}}=0.99$, and significantly slower on invalid trials compared with neutral trials (mean attentional costs $=45 \mathrm{msec}, S D=35 \mathrm{msec}$ ), $t(27)=6.87, p<.001, d_{\mathrm{z}}=1.30$. Figure 3 shows individual and mean RTs in milliseconds per cue condition and hemifield and also illustrates the interindividual variability of attentional benefits (and costs). Results indicate that most participants used the information provided by the spatial cues as intended and performed shifts of attention to the cued hemifield.

\section{Cue-related Brain Activation in the DAN}

The average betas for brain responses observed in each of the four ROIs for leftward and rightward cues compared with neutral cues were all greater than zero. One-sample $t$ tests indicated that the difference from zero was significant for all betas except for beta estimates of activation in response to a rightward cue in the right IPS/SPL (see Table 2) when applying an FDR correction at $q=.05$ (critical $p$ value $=.037$ ). Mean BOLD amplitude changes over the course of 16 TRs $(17.6 \mathrm{sec})$ per cue and ROI are shown in Figure 4 . The $2 \times 2 \times 2$ within-subject ANOVA with factors Cue (left, right), ROI (FEF, IPS/SPL), and Hemisphere (left, right) revealed a significant Cue $\times$ Hemisphere interaction, $F(1,27)=26.32, p<.001$,

Table 2. Means (SDs) and One-sample $t$ Tests with $p$ Values of Betas (Directive - Neutral) per ROI and for Left and Right Cues

\begin{tabular}{llccr}
\hline ROI & Cue & Mean (SD) & \multicolumn{1}{c}{$t$} & \multicolumn{1}{c}{$p$} \\
\hline Left FEF & Left & $.102(.188)$ & 2.860 & .008 \\
& Right & $.179(.194)$ & 4.888 & $<.001$ \\
Right FEF & Left & $.143(.169)$ & 4.492 & $<.001$ \\
& Right & $.071(.163)$ & 2.295 & .030 \\
Left IPS/SPL & Left & $.103(.247)$ & 2.199 & .037 \\
& Right & $.251(.244)$ & 5.441 & $<.001$ \\
Right IPS/SPL & Left & $.141(.225)$ & 3.332 & .003 \\
& Right & $.053(.205)$ & 1.360 & .185 \\
\hline
\end{tabular}


Table 3. Correlation Grouping, Mean Difference between Groups, and $p$ Values per Hypothesis

\begin{tabular}{lllr}
\hline Hypothesis & Expected High & Expected Low & Difference (High - Low) \\
\hline 1. Contralateral & BL-RFEF, BL-RIPS & BL-LFEF, BL-LIPS & 0.049 \\
& BR-LFEF, BR-LIPS & BR-RFEF, BR-RIPS & 0.277 \\
2. Right dominance & BL-RFEF, BL-RIPS & BL-LFEF, BL-LIPS & \\
& BR-LFEF, BR-LIPS & & 0.108 \\
3. Hybrid & BR-RFEF, BR-RIPS & & .217 \\
& BL-RFEF, BL-RIPS & BL-LFEF, BL-LIPS & BR-RFEF \\
\hline
\end{tabular}

Differences between expected high and low correlations are presented for $z$-transformed correlations; $p$ values reflect results of the permutation tests (uncorrected). $\mathrm{BL}=$ left benefits; $\mathrm{BR}=$ right benefits; LFEF = left FEF; RFEF = right FEF; LIPS = left IPS/SPL; RIPS = right IPS/SPL.

$\eta_{\mathrm{p}}^{2}=.494$, for which the difference between left and right cues in the left hemisphere $(-0.113), t(27)=2.48, p=$ $.020, d_{z}=0.47$, was significantly different from and opposite to the difference between left and right cues in the right hemisphere $(0.081), t(27)=1.87, p=.073, d_{z}=0.35$.
This finding is in line with the suggestion that each hemisphere is especially involved in shifts of attention to the contralateral hemifield (see also Figure 4). This was found irrespective of ROI ( $p<.001$ in both ROIs), although there was a trend for a three-way interaction,
Figure 4. Cue-related BOLD signal amplitude changes in the four ROIs. Cues were presented at the onset of Volume 1. Time courses per ROI were baseline-corrected per run. The baseline per run was calculated as the average intensity of the two TRs preceding cue onset across the three cue conditions. Error bars reflect the within-subject standard error adjusted per ROI using the method proposed by Cousineau (2005). The shaded area marks the volumes at which the interaction of cue and hemisphere for neutral-corrected left and right cues was significant at an FDR-corrected threshold of $p=.018(q=.05)$. At Volume 1 , no significant main effects or interactions were found.

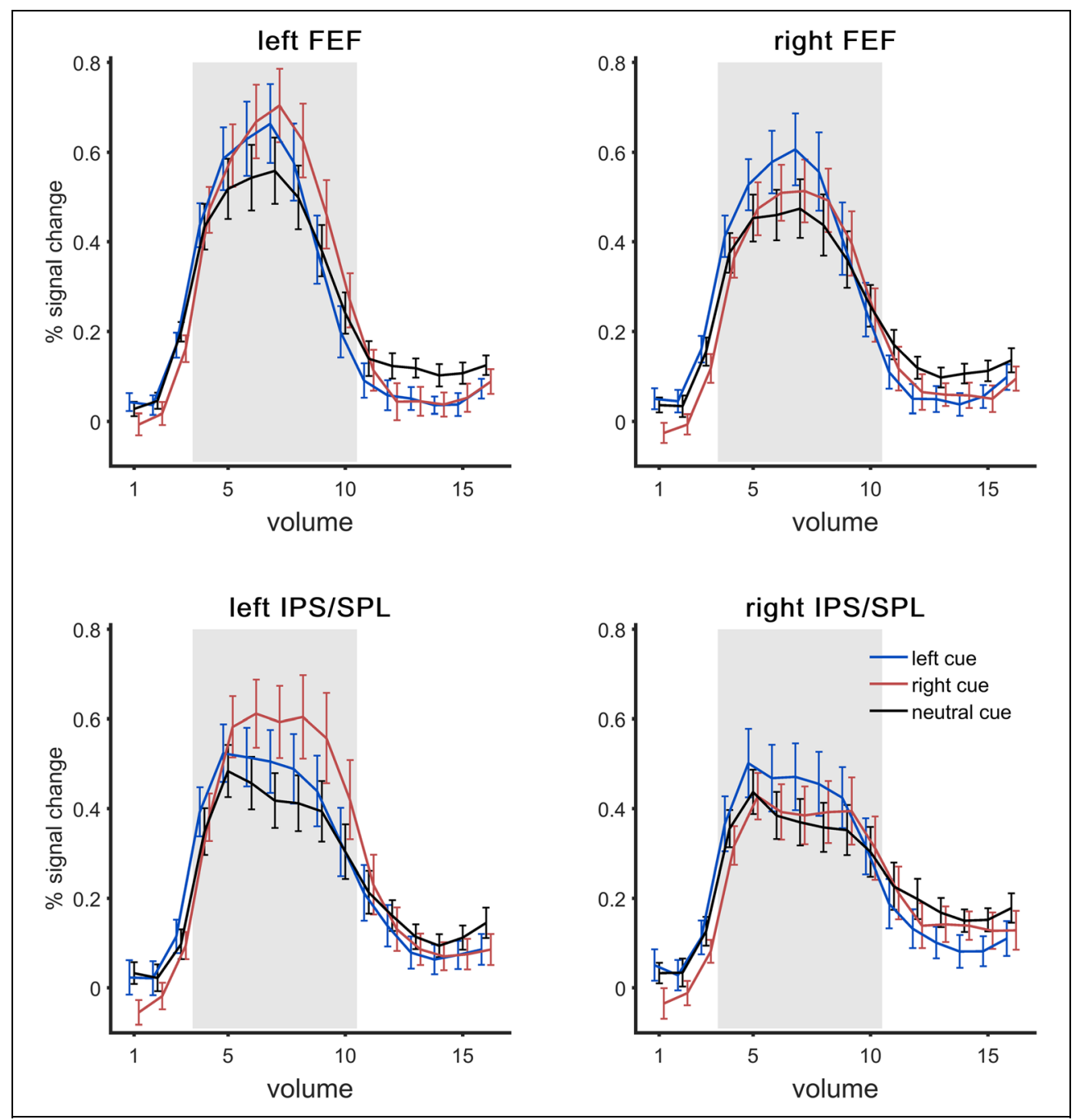


$F(1,27)=3.90, p=.059, \eta_{\mathrm{p}}^{2}=.126$, driven by a stronger Cue $\times$ Hemisphere interaction in IPS/SPL. For illustrative purposes, the time points at which the two-way interaction reached significance also in the analyses of the percent signal change were shaded gray in the plots in Figure 4. We also found a main effect of Hemisphere, $F(1,27)=7.76, p=.010, \eta_{\mathrm{p}}^{2}=.494:$ The left hemisphere showed an overall stronger response to directive cues compared with the right hemisphere. This is possibly due to an overall greater excitability of the left hemisphere because participants responded with their right hand. No other interactions (Cue $\times$ ROI: $F(1,27)=$ $0.73, p=.401, \eta_{\mathrm{p}}^{2}=.026$; ROI $\times$ Hemisphere: $F(1,27)$ $=1.75, p=.198, \eta_{\mathrm{p}}^{2}=.061$ ) or main effects (Cue: $F(1$, $27)=0.16, p=.693, \eta_{\mathrm{p}}^{2}=.006$; ROI: $F(1,27)=0.30, p=$ $\left..587, \eta_{\mathrm{p}}^{2}=.011\right)$ reached significance.

\section{Brain-Behavior Correlations}

Having established that our selected ROIs responded specifically to directive cues and even show contralateral preferences, the central research question was whether the cue-related BOLD responses in the different ROIs could be linked to behavioral effects of covert orienting, that is, attentional benefits in the left and right hemifields, and, more specifically, whether such relationships were hemifield- and hemisphere-specific as existing hypotheses of attention control suggest. In Figure 5, individual benefits in the left and right hemifields are plotted against betas of brain activation in response to left and right cues (compared with neutral cues) for each ROI.

We found significant correlations between brain activation for right cues (compared with neutral cues) and benefits in the right hemifield in all four ROIs (see Figure 5 for Pearson correlation coefficient $r$, uncorrected $p$ values, and 95\% confidence intervals per correlation). For these correlations, individual betas explained up to 23\% of the interindividual variance in attentional benefits. This suggests that frontal and parietal DAN regions in both hemispheres support shifts to the right hemifield, which is in agreement with the right dominance hypothesis. As predicted by all three hypotheses, correlations between benefits in the left hemifield and betas for leftward cues in the left hemisphere were not significant. However, in contrast with all three hypotheses, the correlations between benefits in the left hemifield and activation in response to leftward cues in the right IPS/SPL and right FEF were also not significant, resulting in no significant correlations with benefits in the left hemifield. Note that this also holds when the two extreme values in left benefits (see four left plots in Figure 5) and the very low beta value for left cues in the left IPS/SPL (see lower

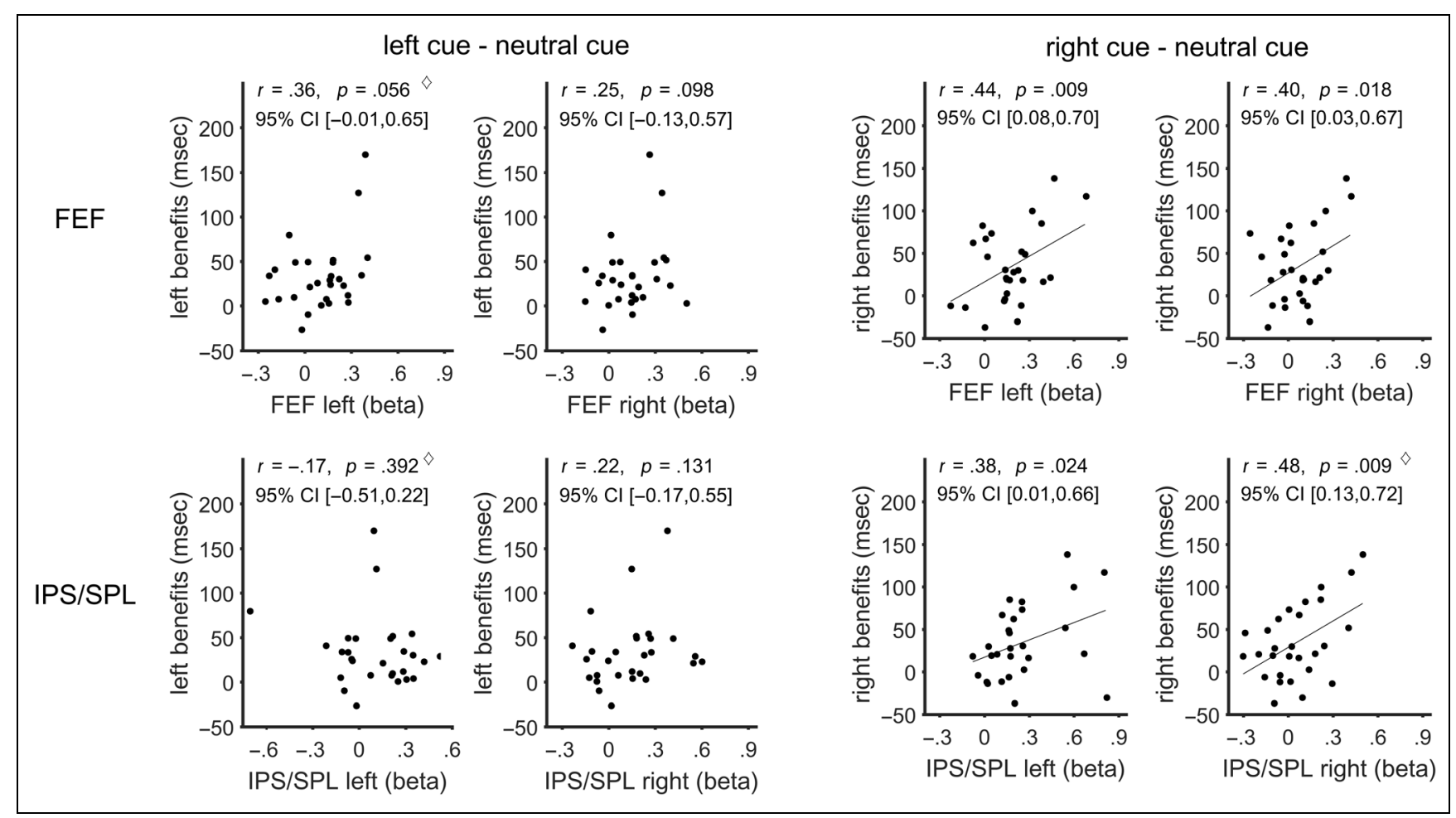

Figure 5. Scatterplots of correlations per ROI between betas for left and right cues and benefits in the corresponding hemifield. Correlations for FEF are displayed at the top; and those for IPS/SPL, at the bottom. Correlations between activation for left cues and left benefits are displayed in the four plots on the left; those between activation for right cues and right benefits are displayed in the four plots on the right. The specified $p$ values are based on two-sided tests for correlations between left benefits and activation in the left FEF and IPS/SPL and the correlation between right benefits and activation in the right IPS/SPL (all marked with a diamond) and are one-sided for all other correlations. All reported confidence intervals are two-sided 95\% confidence intervals, calculated for the $z$-transformed coefficients and then transformed back to $r$ values. Trend lines were added for those correlations surviving an FDR correction at $q=.05$ (critical $p=.024$ ). 


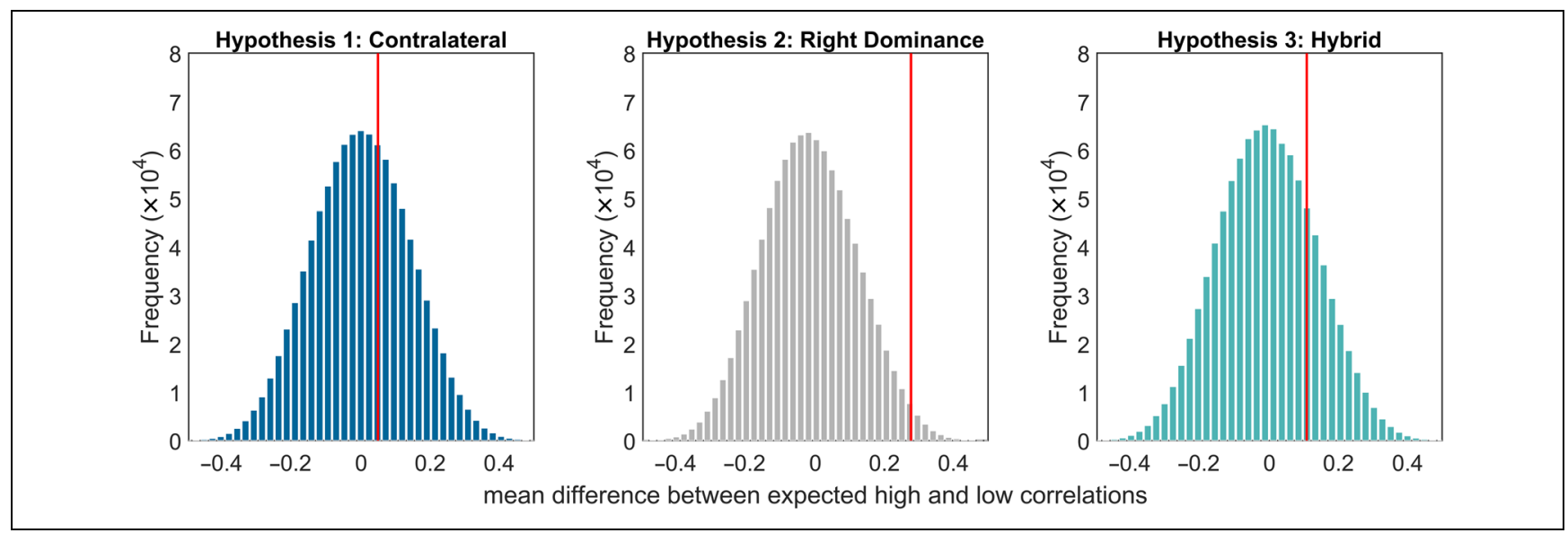

Figure 6. Histograms of permutation tests for the three hypotheses. Distribution of differences between expected high and low correlations under the assumption that all correlations are in fact equal. The red line marks the difference between expected high and low correlations for the observed correlations for each hypothesis.

left plot in Figure 5) are replaced with the nearest value in the remaining sample ( 81 and 82 for benefits in the left hemifield, -.22 for the beta value; $r=.27, .21,-.14$, and .20 for correlations between benefits in the left hemifield and betas in the left FEF, right FEF, left IPS/SPL and right IPS/SPL, respectively).

\section{Permutation Tests}

Finally, we conducted the described permutation tests comparing the expected high and low correlations for each hypothesis and obtained the best fit to the data for the right dominance hypothesis, which proposes that the left as well as the whole right-hemispheric DAN is involved in shifts of attention to the right. More importantly, the permutation test actually revealed a significant difference between the expected low and expected high correlations for the right dominance hypothesis $(p=.015$; Bonferroni-corrected critical $p$ value $=.016$; see Figure 6 and Table 2 for histograms of the generated test distributions and resulting $p$ values for the three hypotheses). The results of the permutation tests also hold when the three outliers discussed in the previous section were replaced in the same manner as described above ( $p$ s $=.362, .012$, and .185 , respectively, in the same order as presented in Table 2 ).

\section{DISCUSSION}

\section{DAN Activation Explains Attentional Benefits}

The first goal of this study was to reveal whether activation related to attention shifts in the DAN, which has been put forth as the neural basis of visuospatial attention control, correlates with effects of selective attention on task performance. We found significant correlations between brain activation parameters specific to directive spatial cues and attentional benefits in mean RTs in the range of $r=.38-.48$. To our knowledge, this is the first demonstration that changes in hemifield-specific visual task performance due to attention can be linked to the cue-related BOLD response in DAN regions in either hemisphere across individuals. Thanks to the inclusion of a neutral cue condition in our task design, we can conclude with fair certainty that the processes we investigated are specific to attention. Both attentional benefits and the betas from the fMRI GLMs that were entered in the correlation analyses were based on difference scores between a condition that required attentional shifting and a condition that did not require attentional shifting but is otherwise comparable in terms of visual input and timing. In addition, the brain activation parameters we used were estimated based on trials without a target (i.e., catch trials), so we can be certain that they reflect purely anticipatory pretarget activation related to the spatially informative cue, independent of target processing and response. This latter improvement in design may also be one of the reasons we managed to find a positive correlation between activation in the nodes of the DAN and attentional benefits, although Small et al. (2003) found no greater activation on trials with large attentional benefits compared with those with no benefits in the DAN when comparing activation for the whole trial, thus not separating target-related from cue-related processes. Finally, our behavioral parameter was more sensitive to gradual effects compared with the binary classification of correct or incorrect responses used in previous studies (Sylvester et al., 2007; Sapir et al., 2005), which might have enabled us to detect the so far elusive link between DAN activation and behavior.

\section{Fit with Hypotheses of Hemispheric Asymmetries}

\section{Right-Hemisphere Activation Explains Variance in Right Benefits}

The second goal of this study was to explore the pattern of observed correlations across the DAN nodes 
and to assess whether this pattern can be explained by any of three existing hypotheses of hemispheric preferences and asymmetries in attention control, namely, the contralateral, right dominance, and hybrid hypotheses. Our permutation tests of the hypotheses suggest that the pattern of correlations fits best with the right dominance hypothesis, and we found a significant difference between the expected high and expected low correlations for this hypothesis only. On the level of the single correlations, we found significant correlations between cue-related activation in all four nodes of the DAN and right benefits (Figure 5, on the right), indicating that the left and right hemispheres are involved in shifts of attention to the right. Supposing that the observed processes in the two hemispheres are at least partially redundant (but see below for a discussion), this can explain the lower frequency and severity of right hemispatial neglect insofar as each hemisphere can compensate for damage in the other hemisphere during attentional shifts to the right hemifield, which is in keeping with the right dominance hypothesis and in conflict with the contralateral hypothesis.

The fact that we found a significant correlation between right benefits and activation in both the righthemisphere FEF and IPS/SPL also argues against the hybrid hypothesis. This hypothesis was derived from findings from brain stimulation studies, which so far found little evidence for a causal involvement of the right IPS in rightward shifts of attention (Duecker \& Sack, 2015). One possible explanation for the discrepancy between our finding and the results of these studies could lie in the fact that the brain stimulation studies often targeted areas that were more posterior and more ventral (often stimulating over electrode P4, e.g., Thut, Nietzel, \& Pascual-Leone, 2005, or even P6, e.g., Fierro et al., 2000) than the ROIs we selected, which extend into SPL. This proposition is supported by Vandenberghe and colleagues, who combined data from different populations and sources of information to dissociate between subprocesses of orienting within the posterior DAN and who concluded that (right) SPL is involved in shifts of attention irrespective of the direction of attention, whereas more posterior IPS/SPL shows hemifield-specific responses (Vandenberghe, Molenberghs, \& Gillebert, 2012). It is therefore conceivable that these stimulation studies targeted a functionally different region than the one we investigated in this study. In line with this notion, Bourgeois, Chica, Valero-Cabré, and Bartolomeo (2013a, 2013b) and Chica, Bartolomeo, and Valero-Cabré (2011) found bilateral effects on inhibition of return, a phenomenon linked to attentional orienting, after stimulating a region in IPS/SPL closer to our own ROIs. Note, however, that inhibition of return is the result of a number of complex processes and that the effects of brain stimulation observed in the study can therefore not be linked unequivocally to a change in orienting performance. It may thus be interesting to systematically target different nodes in the posterior DAN with brain stimulation and to observe and compare the effects on attentional benefits in the left and right hemifields. In an optimal case, this will include functionally defined ROIs, targeted with neuronavigation.

\section{No Neural Correlates of Left Attentional Benefits in the DAN}

Given the good fit between the data and predictions of the right dominance hypothesis and the significant correlations between DAN activation in all four nodes and right benefits, one could conclude that our data support the right dominance hypothesis. However, we did not find significant correlations between brain activation in the right FEF and IPS/SPL and left benefits. On the basis of our data, one would predict no change in leftward orienting after right-hemispheric damage because the right hemisphere is seemingly uninvolved in shifts of attention toward the left hemifield, which is at odds with all three of the tested hypotheses.

Overall, the observed correlations between left benefits and brain activation in the nodes of the DAN were lower compared with those between right benefits and brain activation in the DAN. This suggests that leftward attention shifts are qualitatively different from rightward shifts of attention with regard to how the behavioral effects of orienting are reflected in DAN activation, with a weaker link between cue-related brain activation and behavioral effects for leftward shifts. Assuming that at least the right DAN is involved in shifts of attention to the left, an assumption supported by clinical observations as well as brain stimulation studies, this raises the question why we did not find significant correlations between brain activation in the right DAN and left benefits, although we did find such correlations for right benefits. Speculatively, one possibility is that attentional benefits in the left hemifield are influenced by additional neural processes, which would reduce the fraction of variance explained by DAN activation, leading to lower correlations. Another potential explanation is that shifts to the left require a less drastic change in DAN activation. For example, if the attention system is biased toward the left in healthy participants, as some studies suggest (Jewell \& McCourt, 2000; Bowers \& Heilman, 1980), a shift to the left might require fewer neural resources and might rely less on DAN activation than a shift to the right. Yet, we did not observe lower DAN activation for leftward shifts compared with rightward shifts (no main effect of cued hemifield), and variability in BOLD signal was also comparable for left and right cue trials. Furthermore, others have shown that, although a bias toward the left can be found in the average population, there seem to be observer subtypes with a rightward bias, too (Benwell, Thut, Learmonth, \& Harvey, 2013), which should then also affect the relation between DAN activation and right benefits in a similar manner in those participants, making it less likely that 
the difference between correlations for left and right benefits observed in our study is due to an initial leftward bias of the attention system. As we did not use an independent, validated measure of visuospatial bias such as a line bisection task (Jewell \& McCourt, 2000), we could not test the influence of initial hemifield bias on our results. This remains an opportunity for future studies to investigate.

\section{Ambiguous Evidence for the Contralateral Hypothesis}

The hypothesis least supported by the observed pattern of correlations is the contralateral hypothesis, and its permutation test also yielded the worst fit of the data. At the same time, the ANOVA of the brain response to left and right cues (relative to neutral cues) across regions revealed a significant Hemifield $\times$ Hemisphere interaction and showed that each hemisphere shows at least a trend for a preference for cues to the contralateral hemifield. This is in line with previous findings (Shulman et al., 2010; Sylvester et al., 2007), which were taken as evidence for an assumption of the most prominent model of orienting proposed by Corbetta and Shulman (2002, 2011), namely, that right-hemispheric dominance is not found in the DAN (but rather in a ventral attention network) and that contralateral response patterns are predominant in the DAN. It is thus all the more interesting that our correlation analyses showed that brain activity in response to right cues in the right hemisphere, albeit lower than that in response to left cues, scaled with right benefits, indicating that the cue-related response of the bilateral DAN is informative when it comes to explaining behavioral effects of attentional orienting to the right. This emphasizes how different results can be obtained when looking at different aspects of the brain response in relation to behavior.

\section{Multiple Sources of Interindividual Variability}

Although we convincingly demonstrate a substantial correlation between cue-related brain activation in the DAN and attentional benefits (in the right hemifield), we are limited in the conclusions we can draw about the causes of variability and covariance within and between the two correlated measures. The magnitude of an individual's attentional benefits and the corresponding brain activation may reflect that person's capability to orient attention, including all potential subprocesses such as, for example, disengagement from fixation, recalibration of saliency maps, adaptation of the focality and location of the focus of attention (i.e., the attentional "spotlight" or "gradient"), and maintenance of this state of selective attention. Furthermore, as we are using an endogenous cueing paradigm and are thus investigating voluntary shifts of attention, attentional benefits and cue-related brain responses may also be influenced by a participant's motivation to orient attention, which in turn might depend on various factors such as the general task compliance or the perceived probability of cue validity (although findings suggest that the latter modulates attentional costs rather than benefits; Doricchi, Macci, Silvetti, \& Macaluso, 2010). Finally, because we did not control for eye movements during the MRI data acquisition, some of the variability in the estimated betas and benefits may be due to different levels of compliance with the instruction to fixate. However, previous studies have shown that participants are capable to refrain from moving their eyes in response to the cue or target and that breaks of central fixation occur during a very small percentage of trials only (e.g., Duecker et al., 2013; Doricchi et al., 2010), and we screened participants for their ability to fixate during the training, all in all reducing the likelihood that eye movements influenced our results. To find out which of the remaining factors are driving the observed correlations, it is necessary to use paradigms that isolate the attentional subprocesses and experimentally modify participant-inherent factors, such as motivation, to track resulting changes in the magnitude of the correlations.

\section{Interactions and Possible Nonadditive Processes within the DAN}

As noted already, the hypothesis of right-hemispheric dominance was originally derived to explain the asymmetry in neglect pathology, proposing that an additional involvement of the right hemisphere in shifts to the right hemifield allows for compensation of damage in the left hemisphere, which explains the often less severe symptoms of neglect after lesions in the left hemisphere. This seems to imply some form of redundancy in the neural coding of attention shifts between the two hemispheres. On the other hand, Duecker et al. (2013) found that TMS over the left FEF decreases benefits in the right hemifield, indicating that the contribution from the left FEF is not fully dispensable, which suggests that activation in the different regions reflects additive processes, each contributing to successful shifts of attention. However, an investigation of the additivity or redundancy of the neural responses in the left and right hemispheres is beyond the scope of this study, and studies addressing this will need to account for the fact that all regions of the DAN respond to cues to both hemifields and that measures of brain activation in the different regions thus share a lot of variance (see also Sylvester et al., 2007).

\section{Conclusion}

This study presents new evidence in favor of an involvement of the DAN in effects of selective spatial attention on behavior, showing for the first time that subject-specific measures of DAN activation can explain interindividual variability in attentional benefits in task performance. We found significant correlations between performance changes after shifts of attention to the right (right 
attentional benefits) and BOLD activity in frontal and parietal DAN regions in the left and right hemispheres, in line with the right dominance hypothesis. Although we also found a significant fit between predictions derived from the right dominance hypothesis and the observed pattern of correlations, the pattern of correlations deviates from the predictions in that we found no significant correlations between left benefits and activation in the right DAN, which is in conflict with previous findings from brain stimulation and patient studies and with existing models of spatial attention control. At the same time, we replicated the finding that each hemisphere shows preferential activation for cues to the contralateral hemifield in terms of BOLD signal. Although we thus cannot conclude without reservation that our data support the right dominance hypothesis, the results raise new interesting questions with respect to potential qualitative differences between leftward and rightward shifts of attention and the involvement of different subregions of the (posterior) DAN in different subprocesses of orienting, and they also show that different ways of exploring the data can offer diverging outcomes with respect to the underlying neural mechanisms.

\section{Acknowledgments}

Research was funded by the Netherlands Organization for Scientific Research (NWO 406-13-018 awarded to H. C. M. and A. T. S.; NWO 453-15-008 awarded to A. T. S.) and the European Union's Horizon 2020 research and innovation program under the Marie Sklodowska-Curie grant agreement (no. 708492, awarded to F. D.). We thank Giancarlo Valente, Lourens Waldorp, and Gerard van Breukelen for their help with the statistical aspects of hypothesis testing in the context of our study.

Reprint requests should be sent to Felix Duecker, Faculty of Psychology and Neuroscience, Department of Cognitive Neuroscience, Maastricht University, P. O. Box 616, 6200 MD Maastricht, The Netherlands, or via e-mail: felix.duecker@ maastrichtuniversity.nl.

\section{REFERENCES}

Bartolomeo, P., \& Chokron, S. (1999). Left unilateral neglect or right hyperattention? Neurology, 53, 2023-2027.

Benjamini, Y., \& Hochberg, Y. (1995). Controlling the false discovery rate: A practical and powerful approach to multiple testing. Journal of the Royal Statistical Society, Series B: Methodological, 57, 289-300.

Benwell, C. S., Thut, G., Learmonth, G., \& Harvey, M. (2013). Spatial attention: Differential shifts in pseudoneglect direction with time-on-task and initial bias support the idea of observer subtypes. Neuropsychologia, 51, 2747-2756.

Bourgeois, A., Chica, A. B., Valero-Cabré, A., \& Bartolomeo, P. (2013a). Cortical control of inhibition of return: Causal evidence for task-dependent modulations by dorsal and ventral parietal regions. Cortex, 49, 2229-2238.

Bourgeois, A., Chica, A. B., Valero-Cabré, A., \& Bartolomeo, P. (2013b). Cortical control of inhibition of return: Exploring the causal contributions of the left parietal cortex. Cortex, 49, 2927-2934.
Bowers, D., \& Heilman, K. M. (1980). Pseudoneglect: Effects of hemispace on a tactile line bisection task. Neuropsychologia, 18, 491-498.

Chica, A. B., Bartolomeo, P., \& Valero-Cabré, A. (2011). Dorsal and ventral parietal contributions to spatial orienting in the human brain. Journal of Neuroscience, 31, 8143-8149.

Chica, A. B., Martín-Arévalo, E., Botta, F., \& Lupiáñez, J. (2014). The Spatial Orienting paradigm: How to design and interpret spatial attention experiments. Neuroscience $\mathcal{E}$ Biobehavioral Reviews, 40, 35-51.

Cohen, J. (1973). Eta-squared and partial eta-squared in fixed factor ANOVA designs. Educational and Psychological Measurement, 33, 107-112.

Cohen, J. (1988). Statistical power analysis for the behavioral sciences (2nd ed.). Hillsdale, NJ: Lawrence Erlbaum Associates.

Corbetta, M., \& Shulman, G. L. (2002). Control of goal-directed and stimulus-driven attention in the brain. Nature Reviews Neuroscience, 3, 215-229.

Corbetta, M., \& Shulman, G. L. (2011). Spatial neglect and attention networks. Annual Review of Neuroscience, 34, 569-599.

Cousineau, D. (2005). Confidence intervals in within-subject designs: A simpler solution to Loftus and Masson's method. Tutorials in Quantitative Methods for Psychology, 1, 42-45.

de Haan, B., Morgan, P. S., \& Rorden, C. (2008). Covert orienting of attention and overt eye movements activate identical brain regions. Brain Research, 1204, 102-111.

Desimone, R., \& Duncan, J. (1995). Neural mechanisms of selective visual attention. Annual Review of Neuroscience, 18, 193-222.

Doricchi, F., Macci, E., Silvetti, M., \& Macaluso, E. (2010). Neural correlates of the spatial and expectancy components of endogenous and stimulus-driven orienting of attention in the Posner task. Cerebral Cortex, 20, 1574-1585.

Duecker, F., Formisano, E., \& Sack, A. T. (2013). Hemispheric differences in the voluntary control of spatial attention: Direct evidence for a right-hemispheric dominance within frontal cortex. Journal of Cognitive Neuroscience, 25, 1332-1342.

Duecker, F., \& Sack, A. T. (2015). The hybrid model of attentional control: New insights into hemispheric asymmetries inferred from TMS research. Neuropsychologia, $74,21-29$.

Eickhoff, S. B., Paus, T., Caspers, S., Grosbras, M. H., Evans, A. C., Zilles, K., et al. (2007). Assignment of functional activations to probabilistic cytoarchitectonic areas revisited. Neuroimage, 36, 511-521.

Fierro, B., Brighina, F., Oliveri, M., Piazza, A., La Bua, V., Buffa, D., et al. (2000). Contralateral neglect induced by right posterior parietal rTMS in healthy subjects. NeuroReport, 11, 1519-1521.

Fox, M. D., Corbetta, M., Snyder, A. Z., Vincent, J. L., \& Raichle, M. E. (2006). Spontaneous neuronal activity distinguishes human dorsal and ventral attention systems. Proceedings of the National Academy of Sciences, U.S.A., 103, 10046-10051.

Goebel, R. (2012). BrainVoyager-Past, present, future. Neuroimage, 62, 748-756.

Greenhouse, S. W., \& Geisser, S. (1959). On methods in the analysis of profile data. Psychometrika, 24, 95-112.

Heilman, K. M., \& Valenstein, E. (1979). Mechanisms underlying hemispatial neglect. Annals of Neurology, 5, 166-170.

Heilman, K. M., \& Van den Abell, T. (1980). Right hemisphere dominance for attention: The mechanism underlying hemispheric asymmetries of inattention (neglect). Neurology, 30, 327-330. 
James, W. (1890). The principles of psychology. New York, NY: Henry Holt and Company.

Jenkinson, M., Beckmann, C. F., Behrens, T. E., Woolrich, M. W., \& Smith, S. M. (2012). FSL. Neuroimage, 62, 782-790.

Jewell, G., \& McCourt, M. E. (2000). Pseudoneglect: A review and meta-analysis of performance factors in line bisection tasks. Neuropsychologia, 38, 93-110.

Kastner, S., \& Ungerleider, L. G. (2000). Mechanisms of visual attention in the human cortex. Annual Review of Neuroscience, 23, 315-341.

Kinsbourne, M. (1970). The cerebral basis of lateral asymmetries in attention. Acta Psychologica, 33, 193-201.

Lacadie, C. M., Fulbright, R. K., Constable, R. T., \& Papademetris, X. (2008). More accurate Talairach coordinates for neuroimaging using nonlinear registration. Neuroimage, $42,717-725$.

Lückmann, H. C., Jacobs, H. I. L., \& Sack, A. T. (2014). The cross-functional role of frontoparietal regions in cognition: Internal attention as the overarching mechanism. Progress in Neurobiology, 116, 66-86.

Mauchly, J. W. (1940). Significance test for sphericity of a normal $n$-variate distribution. Annals of Mathematical Statistics, 11, 204-209.

Mesulam, M.-M. (1981). A cortical network for directed attention and unilateral neglect. Annals of Neurology, 10, 309-325.

Nobre, A. C., Gitelman, D. R., Dias, E. C., \& Mesulam, M.-M. (2000). Covert visual spatial orienting and saccades: Overlapping neural systems. Neuroimage, 11, 210-216.

Peirce, J. W. (2008). Generating stimuli for neuroscience using PsychoPy. Frontiers in Neuroinformatics, 2, 10.

Petit, L., \& Haxby, J. V. (1999). Functional anatomy of pursuit eye movements in humans as revealed by fMRI. Journal of Neurophysiology, 82, 463-471.

Posner, M. I. (1980). Orienting of attention. Quarterly Journal of Experimental Psychology, 32, 3-25.
Posner, M. I., Walker, J. A., Friedrich, F. A., \& Rafal, R. D. (1987) How do the parietal lobes direct covert attention?

Neuropsychologia, 25, 135-145.

Sapir, A., D’Avossa, G., McAvoy, M., Shulman, G. L., \& Corbetta, M. (2005). Brain signals for spatial attention predict performance in a motion discrimination task. Proceedings of the National Academy of Sciences, U.S.A., 102, 17810-17815.

Shulman, G. L., Astafiev, S. V., Franke, D., Pope, D. L. W., Snyder, A. Z., McAvoy, M. P., et al. (2009). Interaction of stimulus-driven reorienting and expectation in ventral and dorsal frontoparietal and basal ganglia-cortical networks. Journal of Neuroscience, 29, 4392-4407.

Shulman, G. L., Pope, D. L. W., Astafiev, S. V., McAvoy, M. P., Snyder, A. Z., \& Corbetta, M. (2010). Right hemisphere dominance during spatial selective attention and target detection occurs outside the dorsal frontoparietal network. Journal of Neuroscience, 30, 3640-3651.

Small, D. M., Gitelman, D. R., Gregory, M. D., Nobre, A. C., Parrish, T. B., \& Mesulam, M.-M. (2003). The posterior cingulate and medial prefrontal cortex mediate the anticipatory allocation of spatial attention. Neuroimage, 18, 633-641.

Suchan, J., Rorden, C., \& Karnath, H.-O. (2012). Neglect severity after left and right brain damage. Neuropsychologia, 50, 1136-1141.

Sylvester, C. M., Shulman, G. L., Jack, A. I., \& Corbetta, M. (2007). Asymmetry of anticipatory activity in visual cortex predicts the locus of attention and perception. Journal of Neuroscience, 27, 14424-14433.

Thut, G., Nietzel, A., \& Pascual-Leone, A. (2005). Dorsal posterior parietal rTMS affects voluntary orienting of visuospatial attention. Cerebral Cortex, 15, 628-638.

Vandenberghe, R., Molenberghs, P., \& Gillebert, C. R. (2012). Spatial attention deficits in humans: The critical role of superior compared to inferior parietal lesions. Neuropsychologia, 50, 1092-1103. 